\title{
ON THE EQUIVALENCE OF A TERNARY QUADRATIC FORM
}

E. H. HADLOCK AND AMIN MUWAFI

Introduction. In the classical theory of integral ternary quadratic forms a determinant

$$
d=\left|\begin{array}{lll}
a & t & s \\
t & b & r \\
s & r & c
\end{array}\right| \neq 0
$$

is associated with the form

$$
f=a x^{2}+b y^{2}+c z^{2}+2 r y z+2 s x z+2 t x y .
$$

The g.c.d. of the cofactors of the elements of $d$ is designated by $\Omega$. Then $\Delta$ is defined by $d=\Omega^{2} \Delta$. The concepts of primitive, properly primitive and reciprocal forms as well as equivalence, properties and theorems relating to such concepts are given in [1].

LEMma 1. If $g$ and its reciprocal $G$ are primitive, positive or indefinite forms, then $g$ is equivalent to a primitive form

$$
f=a x^{2}+b y^{2}+c z^{2}+2 r y z+2 s x z+2 t x y
$$

in which

$$
(a, t)=1 \text { and } s \neq 0 .
$$

Proof. Case (i). Let $g$ and $G$ be properly primitive. By [2, Corollary, p. 16] $g$ is equivalent to a form (1) whose first coefficient $a$ and the third coefficient $C$ of whose reciprocal form are positive in tegers which are relatively prime to each other and to $2 \Omega \Delta$. Let $p$ be an odd prime divisor of $a$. Then

$$
(a, C)=(a C, 2 \Omega \Delta)=1 \text { and } \Omega C=a b-t^{2} \Rightarrow p \nmid t .
$$

Thus (2) ${ }_{1}$ holds. If $s=0$, then replacing $x$ by $x+h z, h \neq 0$, in $f$ gives a form in which the coefficient of $2 x z \neq 0$ and such that $a, t$ and $C$ are unchanged.

Case (ii). Let $g$ be improperly primitive and $G$ be properly primitive. By [2, Theorem 20] we may assume that $a / 2$ and $C$ are odd and relatively prime to each other and to $\Omega \Delta$. Thus by $(3)_{3}$

$$
\Omega C=(a / 2)(2 b)-t^{2} \Rightarrow \text { as in Case (i) } p \nmid t .
$$

Received by the editors June 6, 1967. 
Also we observe from the proof of [2, Theorem 19] that $\Omega t$ is odd. Hence (2) 1 holds.

Case (iii). Let $G$ be improperly primitive. By [2, Theorem 21] we may assume that $a$ and $C / 2$ are odd relatively prime integers and prime to $\Delta \Omega$. Thus (3) holds when $C$ is replaced by $C / 2$ and $\Omega$ by $2 \Omega$ and hence $(2)_{1}$ holds.

Leмma 2. There exists a linear transformation $T$ which takes the form $f$ of Lemma 1 into an equivalent form $f_{1}=a x^{2}+b_{1} y^{2}+c_{1} z^{2}+2 r_{1} y z+2 s_{1} x z$ $+2 t_{1} x y$ with the same first coefficient $a$ of $f$ such that the coefficients $s_{1}$ and $t_{1}$ are relatively prime. Moreover the third coefficient $C_{1}$ in $F_{1}$, the reciprocal of $f_{1}$, is equal to $C$ in $F$, the reciprocal of $f$.

Proof. Let $T$ : replace $x$ by $x+k y, y=y, z=z$. Then $s_{1}=s \neq 0, t_{1}$ $=a k+t$. Also $C_{1}=C$ for $\Omega C_{1}=a f(k, 1,0)-t_{1}^{2}=a\left(a k^{2}+b+2 t k\right)-(a k+t)^{2}$ $=\Omega C$.

By (2) and Dirichlet's Theorem, there is an integer $k$ such that $t_{1}=a k+t=p$, a prime and $\left(t_{1}, s_{1}\right)=1$ and where $s_{1} \neq 0$.

Theorem. Every primitive, positive or indefinite, ternary quadratic form is equivalent to a primitive form in which the coefficients of $2 x z$ and $2 x y$ are one and zero respectively.

Proof. Apply the transformation $T_{1}: x=x$, replace $y$ by $s_{1} y+\beta z$ and $z$ by $-t_{1} y+\delta z$ to $f_{1}$ of Lemma 2 to obtain a form $f_{2}$ in which the coefficients $s_{2}$ of $2 x z$ and $t_{2}$ of $2 x y$ are given by

$$
s_{2}=s_{1} \delta+t_{1} \beta, \quad t_{2}=0 .
$$

By Lemma $2,\left(s_{1}, t_{1}\right)=1$ so that there exist integers $\beta$ and $\delta$ such that $s_{2}=1$. Moreover $\left|T_{1}\right|=s_{1} \delta+t_{1} \beta=s_{2}=1$.

REMARK. B. W. Jones has shown that every ternary quadratic form is equivalent to one in which the coefficient of $2 x y$ is zero [3].

\section{REFERENCES}

1. L. E. Dickson, Studies in the theory of numbers, Univ. of Chicago Press, Chicago, Ill., 1930, pp. 6-10.

2. - ibid., Corollary, p. 16; Theorems, 19, 20 and 21, p. 17.

3. B. W. Jones, The regularity of a genus of positive ternary quadratic forms, Trans. Amer. Math. Soc., 33 (1931), 111-124.

4. L. E. Dickson, Modern elementary theory of numbers, Univ. of Chicago Press, Chicago, Ill., 1950; p. 90.

UNIVERSITY OF FLORIDA 